\title{
Metabolic activity of erosions in rheumatoid arthritis
}

\author{
PAULINE PITT, ${ }^{1}$ H BERRY,${ }^{1}$ M CLARKE ${ }^{2}$ H FOLEY, ${ }^{3}$ J BARRATT ${ }^{2}$ \\ AND V PARSONS \\ From the Departments of ${ }^{1}$ Rheumatology, ${ }^{2}$ Nuclear Medicine, ${ }^{3}$ Radiology, and ${ }^{4}$ Nephrology, King's College \\ Hospital, Denmark Hill, London SE5 9RS
}

SUMMARY The hands of 10 patients with rheumatoid arthritis were investigated with diphosphonate scanning and radiology. Increased uptake of isotope can be associated with some erosions but not all and also reflects other processes more linked to acute inflammatory areas unassociated with the development of erosions. If the latter are the hallmark of active rheumatoid arthritis then bone scans are not.

Key words: diphosphonate, radionuclide imaging, radiology.

It is not clear whether rheumatoid erosions ever heal. ${ }^{1-3}$ As erosions might demonstrate variable metabolic activity, we were interested to investigate this possibility by scanning the joints of patients with rheumatoid disease with the bone seeking isotope methylene disphosphonate.

This isotope has been used widely in patients with rheumatoid disease. Some authors ${ }^{4}$ have correlated disease activity with isotope uptake and others ${ }^{5}$ suggest that scans predict radiological outcome. As therapeutic decisions are often based on the appearance of erosions, scans might enable decisions about pharmacological intervention to be taken earlier in the disease process. This study attempts to test these claims and also to investigate the reproducibility of radiographic assessments.

\section{Materials and methods}

Ten patients (five male and five female) with classical or definite rheumatoid arthritis (American Rheumatism Association (ARA) criteria) were investigated. Their age range was 43 to 65 years (average $57 \cdot 4$ years), and the disease duration was $2-47$ years (average 11.3 years). Seven patients were seropositive, three patients were receiving gold, penicillamine, or long term low dose steroids. One was in a drug trial on placebo and the others were on nonsteroidal anti-inflammatory medication-benorylate, salicylate, indomethacin, naproxen, or diclofenac.

\section{Accepted for publication 7 August 1985}

Correspondence to Dr Pauline Pitt, Department of Rheumatology and Rehabilitation, King's College Hospital, Denmark Hill, London SE5 9RS.
Local Ethical Committee approval was obtained for the study.

\section{X - R A Y IN VES T I G A T I ON S}

All patients had conventional posteroanterior (PA) $x$-rays of their hands within 24 hours of diphosphonate scanning. These $x$-rays were examined by two independent observers (a radiologist and a rheumatologist) on two separate occasions. The terminal interphalangeal, proximal interphalangeal, and metacarpophalangeal joints were assessed. Wrists were excluded. If an erosion was seen it was recorded. Each joint was therefore assessed visually on four separate occasions. If on three of these occasions an erosion was seen this designated an erosive joint. $X$-rays were repeated 12 months later and a similar assessment made by the same two observers again on two separate occasions.

\section{CLINICAL ASSESSMENT}

On the day of diphosphonate scanning each patient was examined clinically and a record of the Ritchie articular index, visual analogue pain scale, grip strength, and early morning stiffness was made.

\section{S C A N N I N G}

The instrument used was a model 535 IGE gammacamera interfaced to a system I ADAC nuclear medicine computer system. All recorded images were made in a $128 \times 128$ matrix size, which has a real element size of $4 \times 4 \mathrm{~mm}$. The patient's hands were placed on top of the gammacamera collimator shield in frames with lead separators for each finger. Immediately after the antecubital vein injection of 
$550 \mathrm{MBq}(15 \mathrm{mCi})$ of ${ }^{99 \mathrm{~m}} \mathrm{Tc}-$ methylene diphosphonate data were recorded on a floppy disc in the ADAC system for four minutes, with each frame lasting 10 seconds. This constituted the early scan image. After two to four hours a single 200 second image was recorded similarly; an analogue image was recorded simultaneously for this late scan.

The last minute of the four minute scan was summated. Areas considered to show increased uptake by visual inspection were drawn around with a light pen, and the average number of counts in that area was calculated. The background average counts in the hand was also calculated counting over 10 similarly sized areas and determining the average background counts. Similarly, areas of increased uptake and background measurements were made from the 200 second late scan. To determine whether or not areas of increased uptake, as assessed visually, were quantitatively different from the background at $p<0.05$ the equation shown below was used: ${ }^{6}$

$$
\frac{\mathrm{S}-\mathrm{B}}{\sqrt{(\mathrm{S}+\mathrm{B})}}>2
$$

$\mathrm{S}=$ average count density in that area, $\mathrm{B}=$ background counts per unit area.

If $\mathrm{S}-\mathrm{B}$ over the square root of $\mathrm{S}+\mathrm{B}$ was greater than 2 then the area of increased uptake was significantly different from the background at a $p$ value of less than $0 \cdot 05$. Such areas were considered to show significantly increased uptake of isotope compared with the background.

\section{Results}

\section{X-RAY AS SESSMENT}

The initial radiological assessment yielded 30 erosive joints in five patients. The remaining five had no erosive joints. The reproducibility of $x$-ray observation for each observer between the two occasions when the initial $x$-ray was examined showed observer 1 correlation to be $0 \cdot 6$, observer 2 , correlation to be $0 \cdot 7$. Each observer scored the $x$ rays on two occasions. Therefore, each had two observations on 28 joints in 10 patients, making a total of 560 observations. When the two observers were compared with each other the correlation was $0 \cdot 5$. When scoring repeat $x$-rays taken 12 months later observer 1 correlation was 0.9 , observer 2 correlation was $0 \cdot 8$; when the two observers were compared the correlation was $\mathbf{0 . 7}$. There was no significant difference between observer analysis on either occasion.

SCANNING RESULTS

By determining the statistically significant areas of increased uptake, it was found that $30 \%$ of the joints
Table 1 Areas showing increased uptake of isotope

\begin{tabular}{lr}
\hline Late scan alone & 53 \\
Late and early scan & 14 \\
Early scan alone & 8 \\
Total & 75 \\
\hline
\end{tabular}

Table 2 Behaviour of joints with erosions when scanned with diphosphonate

\begin{tabular}{lr}
\hline No increased uptake of isotope & $13(43 \%)$ \\
Increased uptake on late scan alone & $9(30 \%)$ \\
Increased uptake on both late and early scans & $5(17 \%)$ \\
Increased uptake on early scan alone & $3(10 \%)$ \\
Total & 30 \\
\hline
\end{tabular}

considered positive by visual inspection were not significantly different at $\mathrm{p}<0.05$ to the backround count. In the 10 patients studied, excluding the area of scan corresponding in the wrists, there were 75 areas of increased uptake. In all but two cases these were located over areas corresponding to the $\vec{\oplus}$ proximal interphalangeal and metacarpophalangeal $\%$ joints. The other two areas were over distal interphalangeal joints. These 75 areas showed up on the isotope scans as indicated in Table 1 . Thus of the 75 areas of increased uptake, 67 were seen on the late scan.

There were only 17 scan areas of increased isotope uptake that corresponded to joints with erosions and in all but three of these the scan showed increased uptake during the late scanning periods.

Examination of the behaviour of the 30 erosive joints with diphosphonate scanning showed four $\frac{0}{3}$ different patterns (Table 2).

$43 \%$ of the joints with erosions did not show increased uptake of diphosphonate isotope on either the early or late scan. These results are illustrated in $\mathrm{O}$ Fig. 1, which shows the heterogeneous behaviour of the joints with erosions when scanned with methylene diphosphonate.

When the clinical assessments of these patients $\sigma$ with scanning were correlated there was a significant $N$ correlation $\mathrm{p}<0.01(\mathrm{r}=0.84)$ between the Ritchie $\underset{\mathrm{N}}{\mathrm{N}}$ articular index and the number of areas with 0 increased uptake in the late scan. There was a less significant correlation $(\mathrm{p}<0.05)$ between the late $\frac{}{\Phi}$ scans and the visual analogue scale $(r=0.74)$ and $\stackrel{\oplus}{+}$ grip strength $(r=0.73)$. None of these parameters correlated significantly when the early scans were considered.

The repeat $x$-rays done 12 months later showed 28 new erosions. Thirteen of these occurred in one 


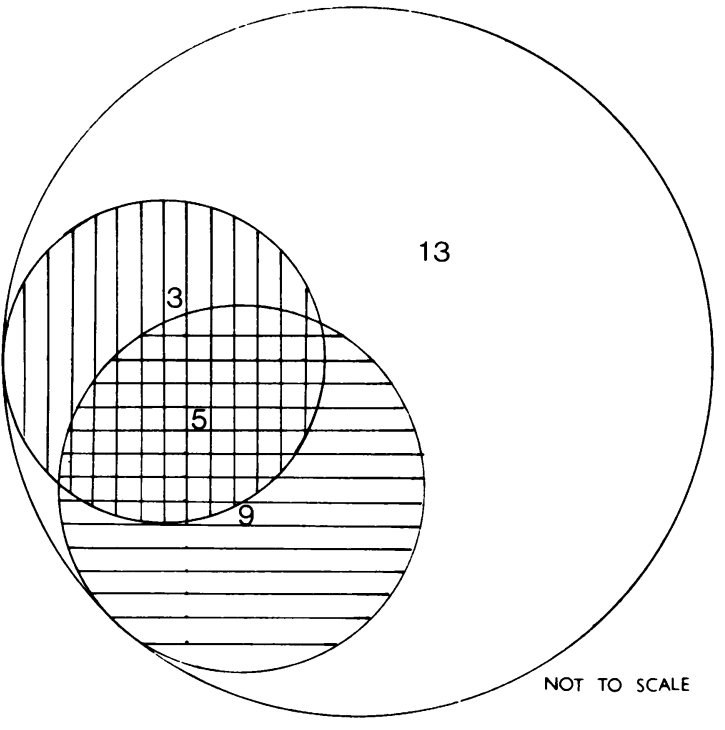

ISOTOPE UPTAKE INCREASE

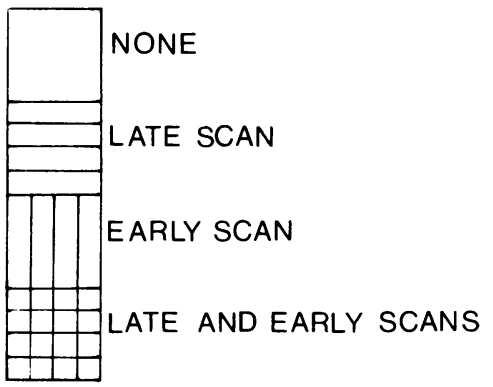

Fig. 1 Behaviour of erosive joints with scanning.

patient. The findings in these joints when scanned 12 months previously is shown in Table 3 . From this it will be seen that over $50 \%$ of the joints with new erosions had not shown increased uptake on either the late or the early scan performed 12 months previously.

Table 3 Behaviour of joints showing new erosions when scanned 12 months previously with diphosphonate

No increased uptake with either scan

Increased uptake on both early and late scans

Increased uptake on late scan only

Increased uptake on early scan only

$15(54 \%)$

$5(18 \%)$

$4(14 \%)$

$4(14 \%)$

Total

\section{Discussion}

Extra bone seeking isotope collection, over and above other areas, is dependent on either increased blood flow or vessel permeability, so that the bone is exposed to a higher concentration of isotope in a given time. It is also dependent on the extra surfaces exposed to the isotope, particularly during acute bone repair ${ }^{7}$ and possibly acute bone destruction. It seems that increased late scanning collection correlated well with an index of inflammation but did not correlate well with the radiological presence of an erosion.

The natural history of erosions, on the other hand, may be due to local inflammatory mediators such as collagenase ${ }^{89}$ and elastase ${ }^{10}$ in association with pannus invasion, humorally mediated bone resorption, ${ }^{11}$ or simple pressure atrophy from the invasion of joint fluid unassociated with an inflammatory or metabolic response.

It is not surprising that joints showing erosions on $x$-ray behave heterogeneously when scanned with a bone seeking isotope, considering the variety of pathological mechanisms that may be involved. We were certainly unable to confirm the suggestion ${ }^{12}$ that erosions always show increased uptake of isotope and found that erosions could appear within 12 months without any preceding bone isotope changes. In many cases the presence of a positive scan was associated with healing of an erosion rather than radiological deterioration as previously suggested. ${ }^{13}$

Although erosions are a hallmark of rheumatoid arthritis, the bone scan only detects some metabolically active erosions and may also reflect more transient vascular and inflammatory changes in bone.

Another feature emerging from this study was the well known difficulty in clearly defining criteria for erosions which would satisfy a number of observers. Internal consistency was high, but cross observer differences were much higher.

\section{References}

1 Fletcher D, Rowley K A. The radiological features of rheumatoid arthritis. $\mathrm{Br} J$ Radiol 1952; 25: 282-95.

2 Soila P. Roentgen manifestations of adult rheumatoid arthritis. Acta Rheumatol Scand 1958: 1-144.

3 Jalava S, Reunanen K. Healing of erosions in rheumatoid arthritis. Scand J Rheumatol 1982; 11: 97-100.

4 Park H M, Telman S A, Ridolfo A S, Wellman H N. A quantative evaluation of rheumatoid arthritic activity with Tc99m HEDP. J Nucl Med 1977; 18: 973-6.

5 Bekerman C, Genant H K. Hoffer P B, Kozin F, Ginsberg M. Radionuclide imaging of the bones and joints of the hand. $A$ definition of normal and a comparison of sensitivity using $99 \mathrm{~m}$ Tc-pertechnetate and 99m Tc-diphosphonate. Radiology 1975; 118: 653-9.

6 Snedecor G W, Cochran W G. Statistical methods. 6th ed. Iowa: Iowa State University Press, 1968: 93. 


\section{Pitt, Berry, Clarke, Foley, Barratt}

7 Savelkoul T J F, Visser W J, Duursma S A. Localisation of ${ }^{99}$ technetium in bone [Abstract]. In: Metals in bone. 1984: 33. (Enlep Symposium, Angers.)

8 Woolley D E. Tetlow L C, Evanson J M. Collagenase at sites of cartilage erosion in the rheumatoid joint. Arthritis Rheum 1977; 20: $1231-9$.

9 Woolley D E, Tetlow L C, Evanson J M. In: Woolley D E, Evanson J M, eds. Collagenase immunolocalisation studies of rheumatoid and malignant tissues, collagenase in normal and pathological connective tissues. Chichester: Wiley, 1980: 105-25.
10 Menninger $H$, Putzier R, Mohr W, Wessinghage R, Tillman K. Granulocyte elastase at the site of cartilage erosion by rheumatoid synovial tissue. J Rheumatol 1980; 39: 145-56.

11 Muriden K D. Periarticular bone lesions in rheumatoid arthritis. Scand J Rheumatol 1975; 4 (suppl 8).

12 Anonymous. Radionuclide bone and joint imaging in rheumatology. Bull Rheum Dis 1979; 30: 1034-9.

13 Weissberg D L, Resnick D, Taylor A, Becker M, Alazraki N Rheumatoid arthritis and its variants. Analysis of scintigraphic radiographic and clinical examinations. Am J Roentgenol 1978; 131: $665-73$. 